\title{
Primary Transglottic Mucosal Melanoma of the Larynx, a Very Rare Entity
}

\author{
Lei Zhou ${ }^{1}$, Maoli Duan ${ }^{2,3}$ and Xinsheng Huang ${ }^{1 *}$ \\ ${ }^{1}$ Department of Otorhinolaryngology-Head and Neck Surgery, Zhongshan Hospital affiliated to Fudan University, China \\ ${ }^{2}$ Department of Clinical Science, Intervention and Technology, Karolinska Institute, Sweden \\ ${ }^{3}$ Department of Otolaryngology Head and Neck Surgery, Karolinska University Hospital, Sweden
}

*Corresponding author: Xinsheng Huang and Maoli Duan, Department of Otorhinolaryngology-Head and Neck Surgery, Zhongshan Hospital affiliated to Fudan University, 180 Fenglin Road, Xuhui, China

To Cite This Article: Lei Zhou, Maoli Duan, Xinsheng Huang. Primary Transglottic Mucosal Melanoma of the Larynx, a Very Rare Entity. 2020 10(1). AJBSR.MS.ID.001475. DOI: 10.34297/AJBSR.2020.10.001475.

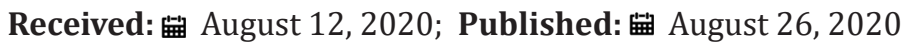

\begin{abstract}
Primary glottic mucosal malignant melanoma of the larynx is a very rare entity with less than 20 cases reported in the literature. The most frequently reported subsite is the supraglottic larynx. The vocal cord as a subsite for primary malignant melanoma is very rare. The present case is a primary transglottic malignant melanoma involving right vocal cord. Mucosal malignant melanoma in the head and neck are historically very aggressive in nature and known for poor outcomes and survival. Most mucosal melanomas described in the literature have been superficial spreading or ulcerative in nature, unlike the present case, in which polypoidal growth was seen with partial dark pigment. Due to the rarity of this entity, every reported case adds to the understanding of its diagnosis and treatment
\end{abstract}

\section{Background}

The primary mucosal melanoma of the larynx is a rare entity [1-3]. Primary non-cutaneous melanomas account for about $1 \%$ of all melanoma cases [4]. About $98 \%$ of tumours of the larynx are of squamous cell histology, $1 \%$ is of glandular origin as adenocarcinomas, and about $0.5 \%$ are rare sarcomas, the malignant melanoma being the medical oddity in this latter group [5]. Owing to rarity of the disease, treatment protocols and prognostic factors are not well established. So, each case is important for understanding the natural course of the disease, tumor morphology and pattern of spread, and for establishing guidelines for further management of the tumor [6]. Until now, there are less than 20 cases, in which have been reported yet [6].

\section{Case Presentation}

A 46 years old woman complained of hoarse throat two years ago, and found a mass on the posterior part of right vocal cord. Then she accepted vocal cord polyp resection. One month ago, she had hoarse throat again, and larygoscopy found the right vocal cord was swelling and immobile (Figure 1). She also has cough, sputum, swallowing obstruction, and breathing obstruction when sleep; sometimes she was wake up due to hard breathing. The patient complaint of no other disease, no family history, and did not drink or smoke. No swelling lymph node was palpated at her neck. The CT scan shows a mass in the larynx, the anterior commissure and the thyroid cartilage is involved. There was no significant finding on her blood test, chest X ray and ultra sound examination of the abdomen. She was married and has children. No skin lesion was found under detailed examination.

\section{Treatment and follow up}

Biopsy showed mucosal melanoma (T4aN0M0, stage IVA, AJCC 8th) [7]. Then total laryngectomy was performed. The resected specimen showed a dark black mass located at the subglottic region (Figure 2), though this typical appearance could not be seen under 
regular laryngoscopy (Figure 1). From the specimen, histological findings showed that the tumor located at the right portion of the larynx, the thyroid cartilage was penetrated, and the extra larynx muscle was involved.

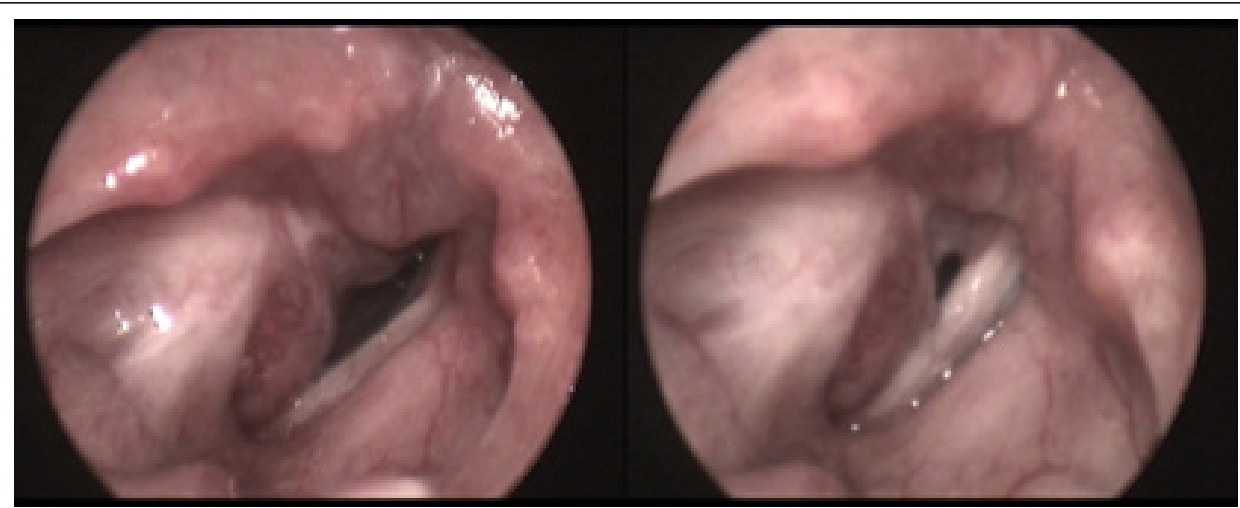

Figure 1: The laryngoscopy of this patient. The right vocal cord could not move and has a swelling appearance, the right false vocal cords also have a swelling appearance.

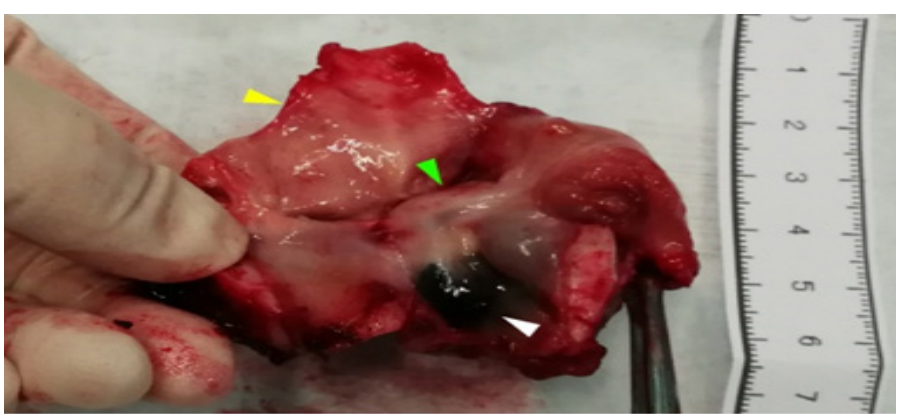

Figure 2: The resected total larynx of this patient. The white arrow indicated the tumor with dark black appearance; the green arrow indicated the swelling right vocal cord; and the yellow arrow was the epiglottis.

The specimen immunohistochemistry finding showed, A103 (-), PNL2 (+), S100 (+), Ki-67 (dense area 60\%+), SOX10 (+), HMB45 $(+)$, p63 (-), PD-L1\{28-8\} (tumor-, interstitial-), PD-L1\{SP142\} (tumor-, interstitial-), PD1(tumor-, interstitial 5\%+), CD117 (weak + ). The B-raf gene examination showed no mutation of the exon 11, and 15 . The $\mathrm{C}$-kit gene examination showed no mutation of the exon [8-15]. The results support the diagnosis of mucosal melanoma of the larynx. No further treatment was prescribed after then. Then she accepted regular follow up, unfortunately, she lost follow up 5 months post operation.

\section{Discussion}

The mucosal melanoma of the larynx is extremely rare. As a result, no definite treatment strategy was established for this rare entity. A systematic review regarding the upper airways tract Table 1: The reported mucosal melanoma cases in the larynx.

\begin{tabular}{|c|c|c|c|}
\hline Cases & Author & Year & Prognosis \\
\hline 1 & Cremonesi [8] & 1956 & no follow up \\
\hline 2 & Welsh and Welsh [5] & 1960 & no follow up \\
\hline 3 & El Barbary et al. [9] & 1968 & Died of disease $(9 \mathrm{~m})$ \\
\hline 4 & El Barbary et al. [9] & 1968 & no follow up \\
\hline
\end{tabular}

mucosal melanoma concluded that surgery followed by radiotherapy improves locoregional control [2]. In the literature, there are only 16 cases of mucosal melanoma in the glottis have been reported yet (Table 1). Due to the rarity of this disease, any case report of the disease is of great important to the diagnoses and treatment. In this case, the patient complained of hoarse throat 2 years ago, and found a polyp located at the posterior portion of the right vocal cord, the resection operation was prescribed with histological result of polyp. However, 2 years later, she complained with hoarse throat again with hard breath, the regular laryngoscopy showed laryngeal mass with right vocal cord immobile. This may indicate that the tumor might initialize from 2 years ago. Additionally, only the subglotic portion of the tumor showed dark black appearance, which cannot be seen under laryngoscope. As a result, thorough histological examination of the disease is crucial. 


\begin{tabular}{|c|c|c|c|}
\hline 5 & Lorentz [10] & 1979 & no follow up \\
\hline 6 & Maki $^{*}$ & 1984 & Died of disease (14 $\mathrm{m}$ ) \\
\hline 7 & Henig [12] & 1989 & no evidence of disease (24m) \\
\hline 8 & Duwel and Michielssen* & 1996 & Died of disease (36 $\mathrm{m}$ ) \\
\hline 9 & Nakanishi et al* & 1996 & no follow up \\
\hline 10 & Alive with disease (10 $\mathrm{m}$ ) \\
\hline 11 & Szmeja et al [14] & 2000 & no evidence of disease (24 $\mathrm{m})$ \\
\hline 12 & Amin et al [15] & 2001 & No details \\
\hline 13 & Aggarwal et al [6] & 2015 & no follow up \\
\hline 14 & Present case & 2020 & Died of disease (4.5 $\mathrm{m})$ \\
\hline 15 & lost follow up (5 $\mathrm{m})$ \\
\hline
\end{tabular}

\section{Declaration of interest}

The authors report no conflicts of interest.

\section{Funding}

This study is supported by Shanghai science and technology committee foundation (17411962200); the Shanghai Pujiang Program (18PJD004) and the National Natural Science Foundation of China (51775547).

\section{References}

1. Carvajal RD, SA Spencer, W Lydiatt (2012) Mucosal melanoma: a clinically and biologically unique disease entity. J Natl Compr Canc Netw, 10(3): 345-356.

2. Jarrom, D, Vinidh Paleri, Cyrus Kerawala, etal. (2017) Mucosal melanoma of the upper airways tract mucosal melanoma: A systematic review with meta-analyses of treatment. Head Neck, 39(4): 819-825.

3. Terada, T, Nobuo Saeki, Kunichika Toh, Nobuhiro Uwa, Kosuke Sagawa, et al. (2007) Primary malignant melanoma of the larynx: a case report and literature review. Auris Nasus Larynx, 34(1): 105-110.

4. Patel SG, Manju L Prasad, Margarita Escrig, Bhuvanesh Singh, Ashok R Shaha, Dennis H Kraus, et al. (2002) Primary mucosal malignant melanoma of the head and neck. Head Neck 24(3): 247-257.

5. Welsh LW, JJ Welsh (1961) Malignant melanoma of the larynx. Laryngoscope 71: 185-191.
6. Aggarwal S, Vivek Kaushal, Sujata Singla, Rajeev Sen (2015) Primary glottic malignant melanoma of the larynx (PGMML): a very rare entity. BMJ case reports 2015:1-4.

7. AJCC, Cancer Staging Manual. $8^{\text {th }}$ edn, 2018.

8. Cremonesi G, (1956) An unusual case of primary melanotic sarcoma of the larynx. Minerva Otorinolaringol 6(6): 333-340.

9. El-Barbary AE, HA Fouad, AF El-Sayed (1968) Malignant melanoma involving the larynx: report of two cases. Ann Otol Rhinol Laryngol 77(2): 338-343.

10. Lorentz E (1979) Malignant melanoma of the larynx (author's transl). HNO 27(8): 275-277.

11. Hussain SS, E Whitehead (1989) Malignant melanoma of the larynx. J Laryngol Otol 103(5): 533-536.

12.Wenig BM (1995) Laryngeal mucosal malignant melanoma. A clinicopathologic, immunohistochemical, and ultrastructural study of four patients and a review of the literature. Cancer 75(7): 1568-1577.

13. Asare-Owusu L, JC Shotton, JB Schofield (1999) Adjuvant radiotherapy for primary mucosal malignant melanoma of the larynx. J Laryngol Otol 113(10): 932-934.

14.Szmeja Z, A Kruk-Zagajewska, J Kaczmarek, L Zieliński, P Majewski (2000) Primary malignant melanoma of the larynx. Otolaryngol Pol 54(2): 173-176.

15. Amin HH, G J Petruzzelli, A N Husain, B J Nickoloff (2001) Primary malignant melanoma of the larynx. Arch Pathol Lab Med 125(2): 271273. 